\begin{tabular}{|c|c|}
\hline Title & Mapping power-law rheology of living cells using multi-frequency force modulation atomic force microscopy \\
\hline Author(s) & Takahashi, Ryosuke; Okajima, Takaharu \\
\hline Citation & $\begin{array}{l}\text { A pplied physics letters, 107(17), } 173702 \\
\text { https://doi.org/10.1063/1.4934874 }\end{array}$ \\
\hline Issue Date & $2015-10-28$ \\
\hline Doc URL & http://hdl.handle.net/2115/60346 \\
\hline Rights & $\begin{array}{l}\text { Copyright } 2015 \text { A merican Institute of Physics. This article may be downloaded for personal use only. A ny other use } \\
\text { requires prior permission of the author and the A merican Institute of Physics. The following article appeared in A ppl. } \\
\text { Phys. Lett. 107, } 173702 \text { (2015) and may be found at } \\
\text { http://scitation.aip.org/content/aip/ ournal/apl/107/17/10.1063/1.4934874 }\end{array}$ \\
\hline Tyре & article \\
\hline File Information & 1.4934874.pdf \\
\hline
\end{tabular}

Instructions for use 


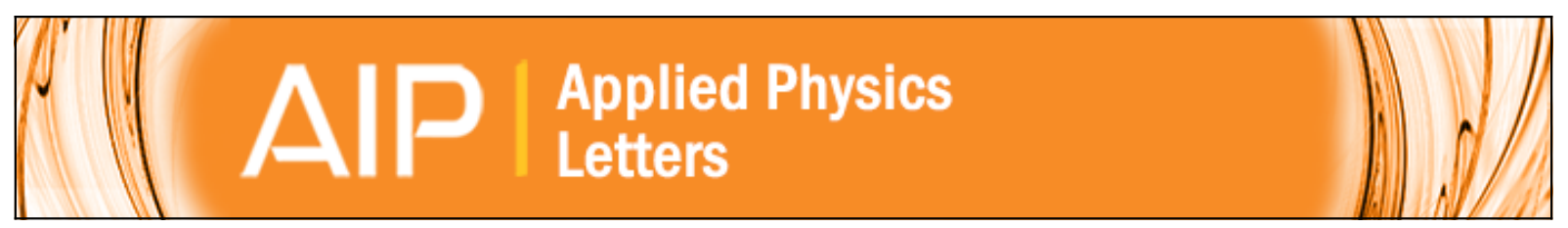

\section{Mapping power-law rheology of living cells using multi-frequency force modulation atomic force microscopy}

Ryosuke Takahashi and Takaharu Okajima

Citation: Applied Physics Letters 107, 173702 (2015); doi: 10.1063/1.4934874

View online: http://dx.doi.org/10.1063/1.4934874

View Table of Contents: http://scitation.aip.org/content/aip/journal/apl/107/17?ver=pdfcov

Published by the AIP Publishing

\section{Articles you may be interested in}

Nanomechanical and topographical imaging of living cells by atomic force microscopy with colloidal probes

Rev. Sci. Instrum. 86, 033705 (2015); 10.1063/1.4915896

Stability enhancement of an atomic force microscope for long-term force measurement including cantilever modification for whole cell deformation

Rev. Sci. Instrum. 83, 093709 (2012); 10.1063/1.4752023

Morphological observation and adhesive property measurement on human ovary carcinoma cells by atomic force microscopy

J. Vac. Sci. Technol. B 27, 1370 (2009); 10.1116/1.3066057

Second harmonic atomic force microscopy of living Staphylococcus aureus bacteria

Appl. Phys. Lett. 94, 043901 (2009); 10.1063/1.3073825

Drift-free atomic force microscopy measurements of cell height and mechanical properties

Rev. Sci. Instrum. 78, 036111 (2007); 10.1063/1.2534889

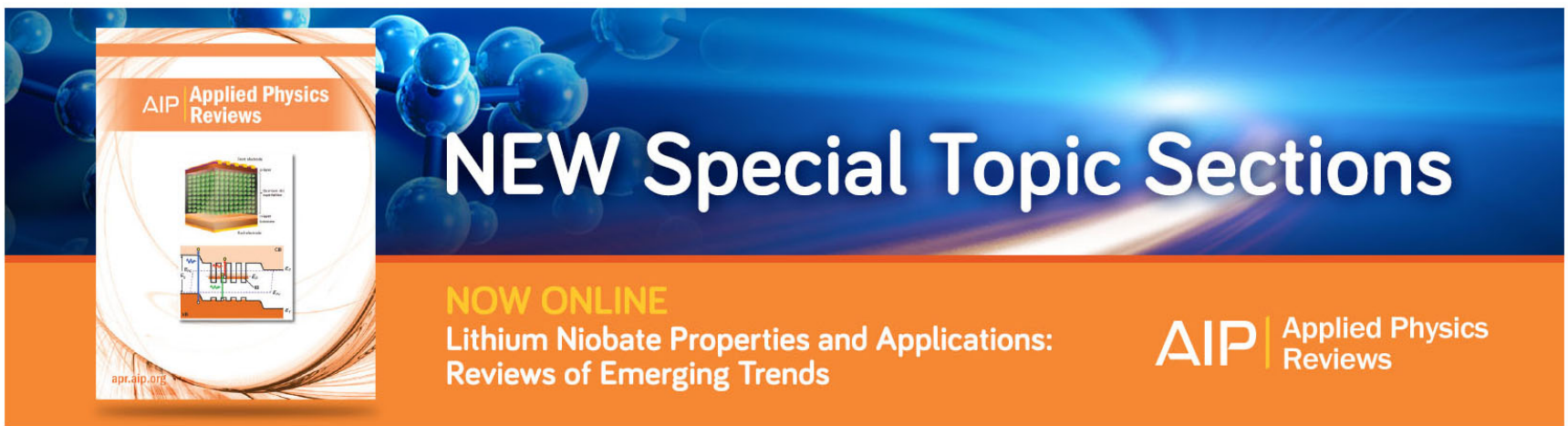




\title{
Mapping power-law rheology of living cells using multi-frequency force modulation atomic force microscopy
}

\author{
Ryosuke Takahashi and Takaharu Okajima ${ }^{\text {a) }}$ \\ Graduate School of Information Science and Technology, Hokkaido University, Kita-ku N14 W9, \\ Sapporo 060-0814, Japan
}

(Received 21 May 2015; accepted 11 October 2015; published online 28 October 2015)

\begin{abstract}
We present multi-frequency force modulation atomic force microscopy (AFM) for mapping the complex shear modulus $G^{*}$ of living cells as a function of frequency over the range of $50-500 \mathrm{~Hz}$ in the same measurement time as the single-frequency force modulation measurement. The AFM technique enables us to reconstruct image maps of rheological parameters, which exhibit a frequency-dependent power-law behavior with respect to $G^{*}$. These quantitative rheological measurements reveal a large spatial variation in $G^{*}$ in this frequency range for single cells. Moreover, we find that the reconstructed images of the power-law rheological parameters are much different from those obtained in force-curve or single-frequency force modulation measurements. This indicates that the former provide information about intracellular mechanical structures of the cells that are usually not resolved with the conventional force measurement methods. (C) 2015 AIP Publishing LLC. [http://dx.doi.org/10.1063/1.4934874]
\end{abstract}

Atomic force microscopy (AFM) has been extensively used for mapping the mechanical properties of living cells. The most conventional technique is the force curve, where the apparent Young's modulus $E$ of a cell can be estimated from the relationship between the indentation depth and the force applied to the cell surface. ${ }^{1,2}$ The method has been extended to mapping other cellular parameters such as deformation, adhesion forces, and dissipation energy. ${ }^{3,4}$

For viscoelastic imaging with AFM, multi-harmonic frequencies near the cantilever resonance were used to acquire elastic and viscous images of cells at high spatial resolution. $^{5,6}$ To evaluate more quantitatively the mechanical properties of compliant cells in a local region, a direct measurement of the cell modulus as a function of frequency is required. Indeed, living cells exhibit characteristic power-law rheological phenomena, i.e., soft, glassy rheology around $10 \mathrm{~Hz} .^{7,8}$ Because this frequency range is much lower than the resonance frequency of the AFM cantilever, an off-resonance measurement is necessary for a detailed understanding of local cellular rheology. One of these AFM techniques is force modulation, in which either the cantilever or the sample is vertically oscillated at a single off-resonant frequency during indentation. The corresponding amplitude and phase shift of the cantilever with respect to the modulation signal are used to estimate the elasticity and viscosity. 9,10 Thus, stepwise changes in the frequency enable an estimate of the frequency-dependent behavior of $G^{*}$ for single cells in a local region. ${ }^{11-13}$

For more detailed understanding of spatial dependence of rheological properties in soft materials, mapping the frequency-dependent rheology of "static" polymer materials ${ }^{14,15}$ and living cells ${ }^{16}$ can be performed by sweeping the frequency during single-frequency force modulation AFM.

\footnotetext{
${ }^{\text {a) }}$ Author to whom correspondence should be addressed. Electronic mail: okajima@ist.hokudai.ac.jp
}

However, because of the extremely long measurement time, this method is not most suitable for living cells that are highly dynamic and continuously remodeling their cytoskeleton. Here, we present multi-frequency force modulation AFM for mapping the frequency dependence of $G^{*}$ for live single cells.

Multi-frequency force modulation is based on the single-frequency version, but now the modulation signal applied to the cantilever during indentation is composed of multiple frequencies (Fig. 1(a)). The procedure here was as follows. At each location in the map, the cell was first indented with a loading force of less than $600 \mathrm{pN}$ without modulation, and the corresponding force curve was used to estimate $E$. Then, during a 0.3 -s indentation, a modulation signal composed of multiple frequencies such as $f=48,80$, $96,120,152,180,200$, and $500 \mathrm{~Hz}$, with an amplitude of ca. $15 \mathrm{~nm}$, was applied to the z-scanner of the cantilever (Fig. $\mathrm{S} 1$ ). The phase of the modulation signal at each frequency was carefully adjusted so that temporal changes in the amplitude of the superimposed signal were minimized (Fig. 1(b)). The amplitude and phase shift of the cantilever deflection with respect to the reference signal were resolved for each frequency using a multiple lock-in amplifier program. In this way, the storage $G^{\prime}$ and loss $G^{\prime \prime}$ moduli were estimated as a function of $f$ (Fig. 1(a)).

To map the cell rheology, the frequency-dependent $G^{\prime}$ and $G^{\prime \prime}$ obtained at each measurement position were fitted to a power-law structural-damping model with additional Newtonian viscosity ${ }^{7,17}$

$$
G *=G_{0} g(\alpha)\{1+i \eta(\alpha)\}\left(\frac{f}{f_{0}}\right)^{\alpha}+i \mu f .
$$

The power-law exponent is $\alpha$ and $g(\alpha)=\Gamma(1-\alpha) \cos (\pi \alpha / 2)$, where $\Gamma$ is the gamma function and $G_{0}$ is a scale factor of the modulus at a frequency-scale factor $f_{0}$, which was 
(a)



(b)

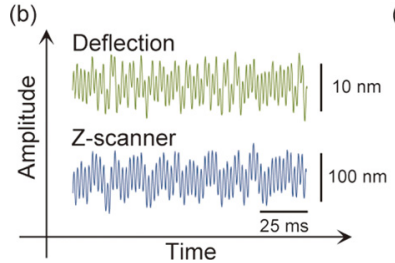

(c)



(d)

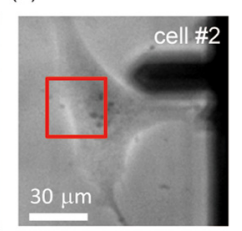

FIG. 1. (a) Schematic of multi-frequency force modulation AFM Modulated voltages with $n$ frequencies from $f=f_{1}$ to $f_{\mathrm{n}}$ are added and applied to the z-scanner to oscillate the cantilever. The amplitude, $A(f)$, and phase shift, $\theta(f)$, of the cantilever deflection signal at each frequency during the indentation were detected with a multiple lock-in amplifier program and used to estimate the storage $G^{\prime}$ and loss $G^{\prime \prime}$ moduli, as a function of $f$ at each measurement position. (b) A modulation signal (blue) and typical deflection signal (green) where the raw deflection signal was subtracted by the slope of the stress relaxation curve observed during the force modulation (see Fig. $\mathrm{S} 1$ ), acquired for $125 \mathrm{~ms}$. Phase contrast images of mouse fibroblast cells \#1 (c) and \#2 (d). The $64 \times 64$ pixel red square in the $30-\mu \mathrm{m}^{2}$ image was the rheology measurement region.

arbitrarily set to $1 \mathrm{~Hz}$. The hysteresivity $\eta(\alpha)$ is equivalent to $\tan (\pi \alpha / 2)$ and $\mu$ is the Newtonian viscous damping coefficient. Further details are in the supplementary material. ${ }^{18}$

Measurements were performed on mouse fibroblast NIH/3T3 (RCB2767, RIKEN) cells \#1 (Fig. 1(c)) and \#2 (Fig. 1(d)). Figures 2(a) and 2(a') show the heights of cells $\# 1$ and \#2, respectively, each of which increases from the periphery to the center where the cell nucleus is located. The apparent Young's modulus $E$ also appeared to gradually increase toward each cell center (Figs. S6(c) and S6 $\left(c^{\prime}\right)$ ). In the $E$ image of cell \#2 (Fig. 2( $\left.\mathrm{b}^{\prime}\right)$ ), vertically aligned structures were observed, which are comparable to the fluorescent

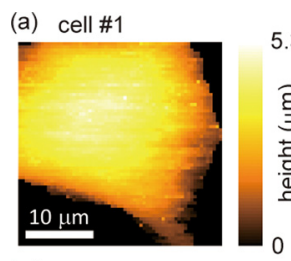

(a') cell \#2

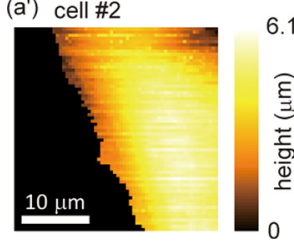

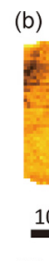

(b')

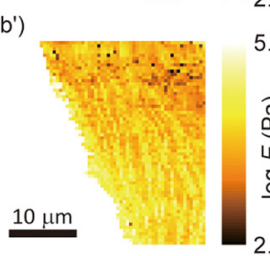

(c)

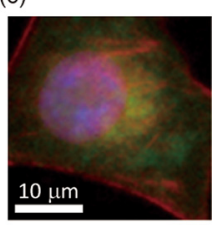

(c')

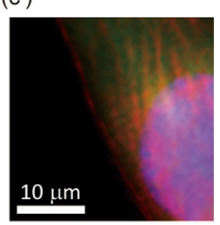

FIG. 2. Height ((a) and $\left.\left(\mathrm{a}^{\prime}\right)\right)$ and apparent Young's modulus $E\left((\mathrm{~b})\right.$ and $\left.\left(\mathrm{b}^{\prime}\right)\right)$ images of cells \#1 and \#2, respectively, estimated from approaching force curves. Immunofluorescence images (c and $\left.c^{\prime}\right)$ of the nucleus (blue), actin filaments (red), and microtubules (green) of cells \#1 and \#2, respectively, in the same region characterized by AFM.

actin filament structures seen in Fig. 2(c'), whereas such filamentous structures were not clearly observed in cell \#1 (Figs. 2(b) and 2(c)). The correlation between $E$ and the actin filaments is consistent with that reported previously. ${ }^{19,20}$

Images of the $G^{\prime}$ and $G^{\prime \prime}$ magnitudes for different frequencies are shown in Fig. 3. We can see that the spatial distributions are quite different between $G^{\prime}$ and $G^{\prime \prime}$, even at the same frequency. For the $G^{\prime}$ images, the magnitude slightly increased with increasing $f$, whereas the spatial distribution was almost constant (Figs. 3(a)-3(h) and $3\left(\mathrm{a}^{\prime}\right)-3\left(\mathrm{~h}^{\prime}\right)$ ). Meanwhile, for the $G^{\prime \prime}$ images, the magnitude increased with $f$, particularly rapidly for $f>150 \mathrm{~Hz}$ (Figs. 3(i) -3 (p) and $\left.3\left(\mathrm{i}^{\prime}\right)-3\left(\mathrm{p}^{\prime}\right)\right)$, and the standard deviation of the spatial distribution of $G^{\prime \prime}$ apparently decreased with $f$ (Fig. S7). The standard deviation of $G^{*}$ measured at different positions quantifies how $G^{*}$ is spatially distributed, and thus it represents the degree of spatial mechanical heterogeneity. This implies that the result shown in Fig. S7 indicates that $G^{\prime}$ was spatially homogeneous at higher frequencies.

Using Eq. (1), we analyzed the data from all the measurement points shown in Fig. 3 and then reconstructed images of the power-law rheological parameters of each cell, as shown in Fig. 4. Comparing Figs. 4(a) and 4(a $\left.{ }^{\prime}\right)$ with Figs. 2(b) and $2\left(\mathrm{~b}^{\prime}\right)$, we notice that the $G_{0}$ image is qualitatively similar to the $E$ images for cells \#1 and \#2. This is because $E$ measured by an AFM force curve involves the elastic component averaged in a broad frequency range, which depends on the approach rate of force curve measurement.

The image of $\alpha$ is significantly different from that of $E$ and $G_{0}$ (Figs. 4(b) and $4\left(\mathrm{~b}^{\prime}\right)$ ). Surprisingly, for cell \#1 (Fig. 4(b)), $\alpha$ drastically decreases toward the cell center where the cell nucleus is located, as shown in Fig. 2(c). This indicates that the $\alpha$ image provides information about intracellular mechanical structures that are usually not resolved in force-curve and single-frequency force modulation methods.

It has been reported that the images relating to intracellular nuclear structures have been observed with ultrasound holographic AFM imaging, in which the sample and the cantilever are oscillated at slightly different ultrasound frequencies, ${ }^{21}$ although the mechanism for ultrasonic holographic AFM imaging is not fully understood. Figure 4(b) suggests that the mapping rheological parameters via force modulation AFM can reveal intracellular structures.

The decrease in $\alpha$ over the nucleus of cell \#1 shown in Figs. 4(b) and S6(e) is similar to the ensemble-averaged behavior measured in single cells attached to the patterned substrates. ${ }^{22}$ This indicates that cells are likely to be close to a purely elastic material, ${ }^{7,8,17}$ which could be a solid-like actin filamentous structure over the nucleus. In contrast, such an intracellular structure was not observed in cell \#2, and $\alpha$ tended to slightly increase toward the cell center (Figs. 4(b') and $\left.\mathrm{S} 6\left(\mathrm{e}^{\prime}\right)\right)$. It is widely recognized that $\alpha$ is intimately associated with remodeling of the cytoskeleton, especially actin filaments. ${ }^{8}$ Thus, the spatial difference in $\alpha$ over the cell nucleus seen in Figs. 4(b) and $4\left(b^{\prime}\right)$ implies that dynamic behavior of actin filaments over the cell nucleus can vary considerably among cells.

A comparison between Figs. 2(c) and 2(c') and Figs. 4(c) and $4\left(\mathrm{c}^{\prime}\right)$ reveals that $\mu$ slightly increased toward the cell nucleus where the concentration of microtubules is high 




FIG. 3. $G^{\prime}$ and $G^{\prime \prime}$ images measured in cell \#1 ((a)-(p)) and cell \#2 $\left(\left(\mathrm{a}^{\prime}\right)-\left(\mathrm{p}^{\prime}\right)\right)$, respectively, at 48, 80, 96, 120, 152, 180, 200, and 500 Hz.

and decreased in the regions away from the cell nucleus where the microtubule concentration is lower (Figs. S6(f) and $S 6\left(f^{\prime}\right)$ ). This result implies that crowded microtubule networks affect the fluidity that is attributed to the Newtonian viscosity.

Figures 5(b) and 5(b') show the averaged $G^{\prime}$ and $G^{\prime \prime}$ of cells \#1 and \#2, respectively, in regions close to and far away from the cell nucleus, which are depicted in Figs. 5(a) and $5\left(\mathrm{a}^{\prime}\right)$. The averaged $G^{\prime}$ and $G^{\prime \prime}$ are well fit by Eq. (1) for both cells, but the magnitudes are largely different between the cells, indicating that there is a large variation in $G^{*}$ within living cells. ${ }^{13}$ Moreover, a linear relationship between $\log G_{0}$ and $\alpha$, which was observed previously in ensembles, ${ }^{7,13,17}$ appeared for cell \#1 (Fig. 5(c)). However, those values exhibited no correlation for cell \#2 (Fig. 5( $\left(^{\prime}\right)$ ). This indicates that the linear relation of $\log G_{0} v s . \alpha$ is not necessarily satisfied at the single-cell level.

Rheological properties of single cells have been investigated in the time domain with creep ${ }^{23,24}$ and stress relaxation $^{25-27}$ experiments. Those techniques were used recently to map the relaxation behavior of single cells. ${ }^{28,29}$ Because of the finite time lag between contact and measurement,

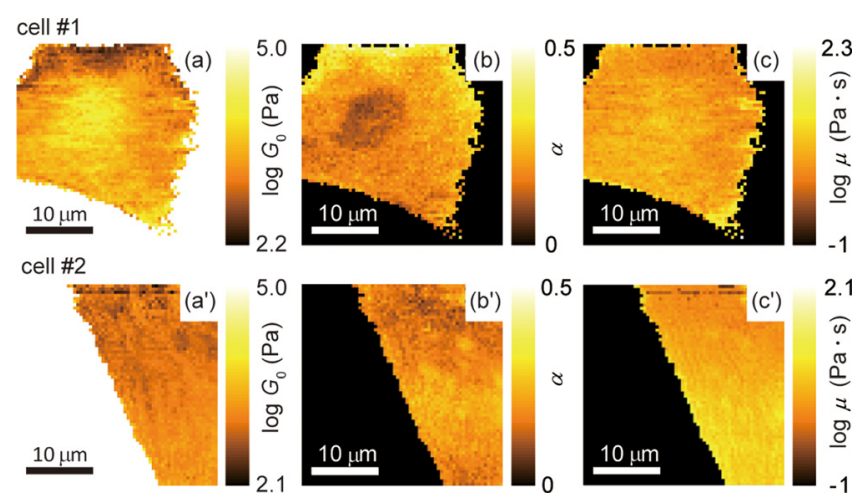

FIG. 4. Images of single power-law rheological parameters of $G_{0}$ ((a) and $\left.\left(\mathrm{a}^{\prime}\right)\right), \alpha\left((\mathrm{b})\right.$ and $\left.\left(\mathrm{b}^{\prime}\right)\right)$, and $\mu\left((\mathrm{c})\right.$ and $\left.\left(\mathrm{c}^{\prime}\right)\right)$ in cells \#1 and \#2. however, it is difficult to monitor the instantaneous change in the deflection signal just after contact. This suffers from the precise estimate of the Newtonian viscous damping coefficient $\mu$ in the power-law rheology. ${ }^{17}$ Also, to optimize the mapping time, a longer relaxation process exhibiting a power-law behavior ${ }^{24}$ is difficult to be measured. ${ }^{28,29}$

Those limits mentioned above are not imposed on frequency domain measurements. In the present study, multi-frequency force modulation AFM measurements were conducted over two orders of magnitude in frequency, which was limited at the high end only by the piezo-induced mechanical vibration of the cantilever. The frequency bandwidth may be extended by using magnetic vibration, ${ }^{30}$ which would more precisely estimate $\mu$.

To verify the accuracy of multi-frequency force modulation AFM presented here, we conducted a mapping experiment of a "static" silicone polymer substrate. We confirmed that the frequency-dependence of $G^{*}$ measured in this multi-frequency force modulation AFM was consistent with that measured in a step-wise change in frequency in the single-frequency force


(c')

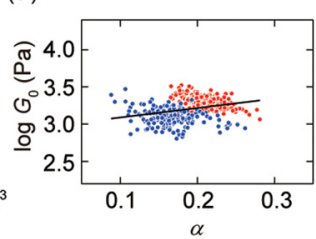

FIG. 5. Average storage $G^{\prime}$ (filled circle) and loss $G^{\prime \prime}$ (open circle) moduli (b and $b^{\prime}$ ) of cell \#1 (a) and cell \#2 ( $\left.a^{\prime}\right)$, the images of which are the maps of $E$, in the regions over (red) and away from (blue) the cell nucleus. Solid lines represent fits with Eq. (1). The relationship of $\log G_{0}$ vs. $\alpha$ in cell \#1 (c) and cell \#2 ( $\left.\mathrm{c}^{\prime}\right)$. Solid lines represent fits with a linear function of $\log G_{0}$ vs $\alpha$. 
modulation AFM (Fig. S4). Moreover, the spatial distributions of rheological parameters of the silicone substrate were almost identical for the successive mapping images (Fig. S5). These results suggest that the multi-frequency force modulation AFM is applicable to various soft materials, and the mapping images are highly reproducible.

In summary, multi-frequency force modulation AFM enabled the measurement of the complex shear modulus, in a force-mapping manner, of single cells as a function of frequency. The method is off-resonance, and thus, differs from cantilever-resonance multi-frequency AFM. ${ }^{31}$ Images of the power-law exponent exhibited spatial variations that were much different from the images of elastic modulus obtained in the force-curve or single-frequency force modulation measurements. It is anticipated that extending the bandwidth in force modulation experiments for living cells with a magnetic force technique ${ }^{30}$ will achieve a high-speed mapping.

The study was supported in part by Grants-in-Aid for Scientific Research (B) (25286081), a Grant-in-Aid for Scientific Research on Innovative Areas "Bio-assembler" (26106701) and "Fluctuation and Structure" (26103501), and a Grant-in-Aid for challenging Exploratory Research (15K13393).

${ }^{1}$ E. A.-Hassan, W. F. Heinz, M. D. Antonik, N. P. D'Costa, S. Nageswaran, C. A. Schoenenberger, and J. H. Hoh, Biophys. J. 74, 1564 (1998).

${ }^{2}$ C. Braunsmann, J. Seifert, J. Rheinlaender, and T. E. Schäffer, Rev. Sci. Instrum. 85, 073703 (2014).

${ }^{3}$ Y. F. Dufrêne, D. Martinez-Martin, I. Medalsy, D. Alsteens, and D. J. Müller, Nat. Methods 10, 847 (2013).

${ }^{4}$ C. Heu, A. Berquand, C. Elie-Caille, and L. Nicod, J. Struct. Biol. 178, 1 (2012).

${ }^{5}$ A. Raman, S. Trigueros, A. Cartagena, A. P. Stevenson, M. Susilo, E. Nauman, and S. A. Contera, Nat. Nanotechnol. 6, 809 (2011).

${ }^{6}$ A. Cartagena and A. Raman, Biophys. J. 106, 1033 (2014).

${ }^{7}$ B. Fabry, G. N. Maksym, J. P. Butler, M. Glogauer, D. Navajas, and J. J. Fredberg, Phys. Rev. Lett. 87, 148102 (2001).

${ }^{8}$ P. Kollmannsberger and B. Fabry, Annu. Rev. Mater. Res. 41, 75 (2011).
${ }^{9}$ M. Radmacher, R. W. Tillmann, M. Fritz, and H. E. Gaub, Science 257, 1900 (1992).

${ }^{10}$ M. Radmacher, R. W. Tilmann, and H. E. Gaub, Biophys. J. 64, 735 (1993).

${ }^{11}$ J. Alcaraz, L. Buscemi, M. Grabulosa, X. Trepat, B. Fabry, R. Farré, and D. Navajas, Biophys. J. 84, 2071 (2003).

${ }^{12}$ S. Hiratsuka, Y. Mizutani, M. Tsuchiya, K. Kawahara, H. Tokumoto, and T. Okajima, Ultramicroscopy 109, 937 (2009).

${ }^{13}$ P. Cai, Y. Mizutani, M. Tsuchiya, J. M. Maloney, B. Fabry, K. J. Van Vliet, and T. Okajima, Biophys. J. 105, 1093 (2013).

${ }^{14}$ T. Igarashi, S. Fujinami, T. Nishi, N. Asao, and K. Nakajima, Macromolecules 46, 1916 (2013).

${ }^{15}$ H. K. Nguyen, M. Ito, S. Fujinami, and K. Nakajima, Macromolecules 47, 7971 (2014).

${ }^{16}$ J. Rother, H. Noding, I. Mey, and A. Janshoff, Open Biol. 4, 140046 (2014).

${ }^{17}$ B. Fabry, G. N. Maksym, J. P. Butler, M. Glogauer, D. Navajas, N. A. Taback, E. J. Millet, and J. J. Fredberg, Phys. Rev. E 68, 041914 (2003).

${ }^{18}$ See supplementary information at http://dx.doi.org/10.1063/1.4934874 for experimental details and additional results.

${ }^{19}$ C. Rotsch, K. Jacobson, and M. Radmacher, Proc. Natl. Acad. Sci. U.S.A. 96, 921 (1999).

${ }^{20}$ C. Rotsch and M. Radmacher, Biophys. J. 78, 520 (2000).

${ }^{21}$ L. Tetard, A. Passian, K. T. Venmar, R. M. Lynch, B. H. Voy, G. Shekhawat, V. P. Dravid, and T. Thundat, Nat. Nanotechnol. 3, 501 (2008).

${ }^{22}$ C. Y. Park, D. Tambe, A. M. Alencar, X. Trepat, E. H. Zhou, E. Millet, J. P. Butler, and J. J. Fredberg, Am. J. Phys. 298, C1245 (2010).

${ }^{23}$ H. W. Wu, T. Kuhn, and V. T. Moy, Scanning 20, 389 (1998).

${ }^{24}$ S. Hiratsuka, Y. Mizutani, A. Toda, N. Fukushima, K. Kawahara, H. Tokumoto, and T. Okajima, Jpn. J. Appl. Phys., Part 1 48, 08JB17 (2009).

${ }^{25}$ E. M. Darling, S. Zauscher, and F. Guilak, Osteoarthr. Cartil. 14, 571 (2006).

${ }^{26}$ E. M. Darling, S. Zauscher, J. A. Block, and F. Guilak, Biophys. J. 92, 1784 (2007).

${ }^{27}$ T. Okajima, M. Tanaka, S. Tsukiyama, T. Kadowaki, S. Yamamoto, M. Shimomura, and H. Tokumoto, Nanotechnology 18, 084010 (2007).

${ }^{28}$ S. Moreno-Flores, R. Benitez, M. D. Vivanco, and J. L. Toca-Herrera, J. Biomech. 43, 349 (2010).

${ }^{29}$ F. M. Hecht, J. Rheinlaender, N. Schierbaum, W. H. Goldmann, B. Fabry, and T. E. Schäffer, Soft Matter 11, 4584 (2015).

${ }^{30}$ L. M. Rebelo, J. S. de Sousa, J. M. Filho, J. Schape, H. Doschke, and M. Radmacher, Soft Matter 10, 2141 (2014).

${ }^{31}$ R. Garcia and E. T. Herruzo, Nat. Nanotechnol. 7, 217 (2012). 